\title{
DEVELOPMENT OF UAV PHOTOGRAMMETRY METHOD BY USING SMALL NUMBER OF VERTICAL IMAGES
}

\author{
Y. Kunii* \\ Department of Landscape Architecture Science, Tokyo University of Agriculture \\ 1-1-1 Sakuragaoka, Setagaya-ku, Tokyo, 156-8502, Japan \\ y3kunii@nodai.ac.jp
}

Commission II, ICWG I/II

KEY WORDS: Vertical Image, UAV, Camera Calibration, Test Site, Relative Orientation

\begin{abstract}
:
This new and efficient photogrammetric method for unmanned aerial vehicles (UAVs) requires only a few images taken in the vertical direction at different altitudes. The method includes an original relative orientation procedure which can be applied to images captured along the vertical direction. The final orientation determines the absolute orientation for every parameter and is used for calculating the 3D coordinates of every measurement point. The measurement accuracy was checked at the UAV test site of the Japan Society for Photogrammetry and Remote Sensing. Five vertical images were taken at 70 to $90 \mathrm{~m}$ altitude. The 3D coordinates of the measurement points were calculated. The plane and height accuracies were $\pm 0.093 \mathrm{~m}$ and $\pm 0.166 \mathrm{~m}$, respectively. These values are of higher accuracy than the results of the traditional photogrammetric method. The proposed method can measure 3D positions efficiently and would be a useful tool for construction and disaster sites and for other field surveying purposes.
\end{abstract}

\section{INTRODUCTION}

The demand for the unmanned aerial vehicles (UAVs) is increasing as they find applications in various fields. For example, more accurate geographical data can be acquired by using the UAVs than by using the usual aerial photogrammetry (Valavanis and Vachtsevanos, 2015). The UAVs can take high resolution images as they are able to fly at low altitudes (Beaudoin et al., 2015). In addition, the UAVs can be used for observation of natural disasters (Galarreta et al., 2015 and Li et al., 2012) or for surveying the construction sites (Barazzetti et al., 2014 and Feifei et al., 2012). Such applications need rapid and low-cost surveying, and the UAVs are well suited for that purpose (Tanzi et al., 2016). In order to acquire geographical data by aerial photogrammetry, many images should be taken from an aerial vehicle which moves in horizontal direction and at a fixed altitude (Amrullah et al., 2016). After that, the images are processed with the help of the structure-from-motion (SfM) technique (Westoby et al., 2012). Multiple neighbouring images with a high rate of overlapping should be obtained for high accuracy measurement (Bagheri et al., 2015), which calls for labour and cost. In the event of natural disasters, UAV operation may sometimes involve risk (Longhitano and Quintanilha, 2013) and should be avoided. Therefore, an easy and convenient method of operating the UAVs is strongly needed. Reports exist on some applications of the UAVs with other devices (Persad and Armenakis, 2016), but it will be difficult to prepare a number of such devices in emergency.

We developed a new camera calibration and measurement method which requires only a few images taken in a simple UAV flight. The UAV in this method was flied vertically and the images were taken at a different altitude. We compared the measurement accuracy of the proposed method against the SfM method and evaluated the performance of the proposed method by checking the accuracy.

\section{ACQISITION OF IMAGES FOR EVALUATION}

The images for checking the accuracy were taken at a UAV test site in Kanagawa, Japan. The UAV test site is managed by the Japan Society for Photogrammetry and Remote Sensing. Figure 1 shows the entrance to the UAV test site. There are 76 points of circular ground marks in the test area of about $5,000 \mathrm{~m}^{2}$, as shown in figure 2. The coordinates of the points are given in Japanese Geodetic Datum 2000 (JGD2000) coordinate system. The centre coordinates of the ground marks were given by performing the ground survey of the whole site by a total station. This allowed to compare the given coordinates and the results of the UAV photogrammetry and check the accuracy of the photogrammetry. Figure 3 shows the UAV "DJI Inspire 1" which was used for taking the images. The camera "FC350" on the Inspire 1 has

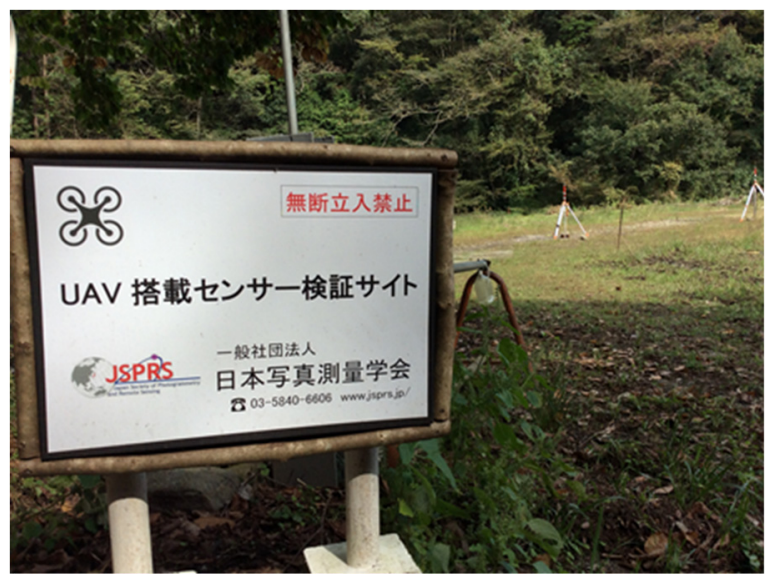

Figure 1. Entrance to UAV test site

\footnotetext{
* Corresponding author
} 


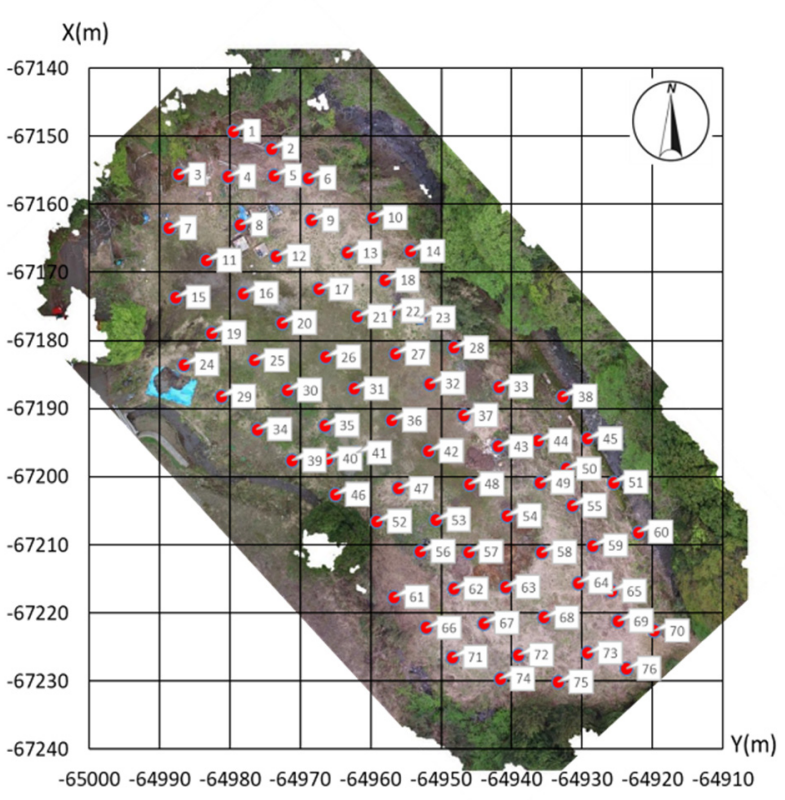

Figure 2. 76 ground marks in test site

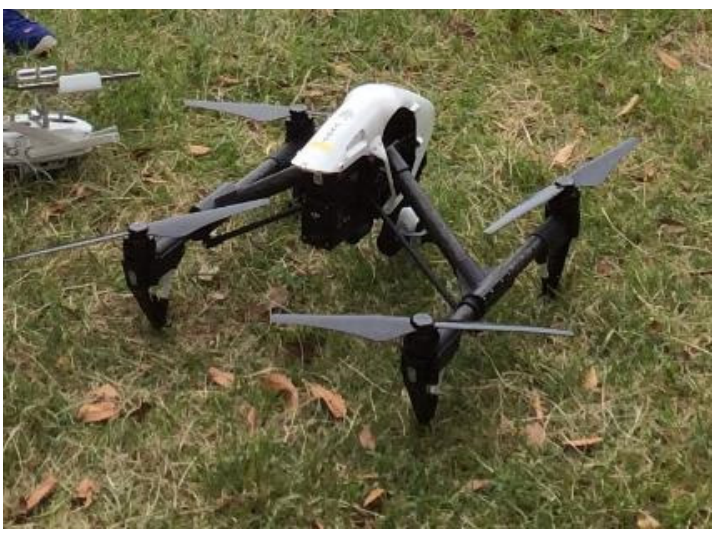

Figure 3. DJI Inspire 1

$4,000 \times 2,250$ pixels and $4 \mathrm{~mm}$ focal length. Figure 4 shows the picture of the overall test site taken by Inspire 1 .

The proposed method can measure the $3 \mathrm{D}$ coordinates without the ground control points (GCPs). However, one distance is needed as a given value. Therefore, the distance between point No. 27 and No. $35(14.831 \mathrm{~m})$ was used for the method, as shown in figure 5 .

\section{THEORY OF THE PROPOSED METHOD}

The proposed method requires vertical images. Some corresponding points on the vertical images were extracted, and the camera calibration and 3D measurement were performed simultaneously. The details of the method are as follows.

\subsection{Relative Distance Between Principal Points}

For taking vertical images, a relative position of each principal point was estimated. Figure 6 shows the positional relation of the vertical images. The figure also shows the altitudes for each image and the approximate value for each altitude. The given distance between two points is $L$; the given distance $L$ on sensors is $l_{1}, l_{2}, \ldots$ and $l_{5}$. Thus, the approximate altitudes $H_{1}, H_{2}, \ldots$ and $\mathrm{H}_{5}$ could be calculated by the following equation.

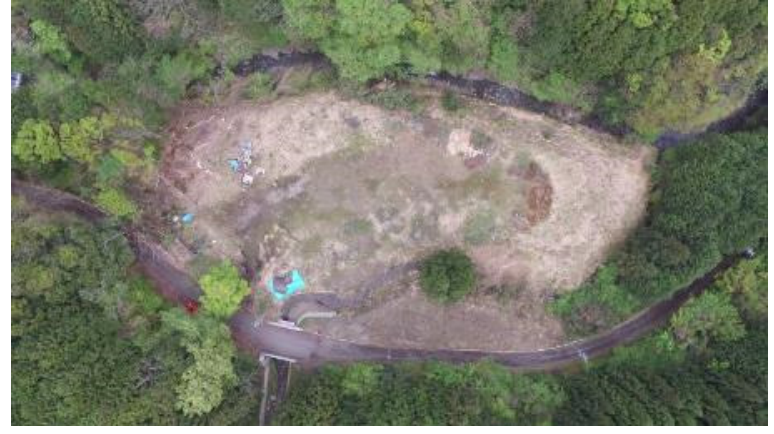

Figure 4. UAV test site taken by Inspire 1

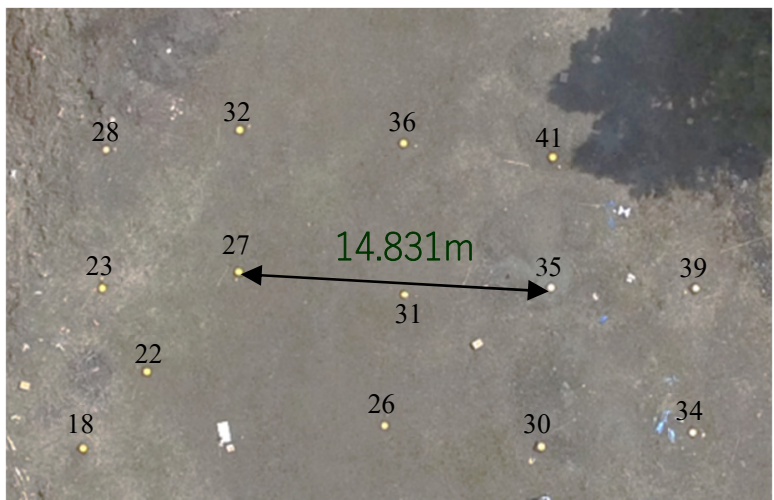

Figure 5. Given distance between 2 points

$H_{i}=\frac{L}{l_{i}} f \quad(i=1,2,3, \Lambda)$

where,

$H_{i}$ : Altitude of pictures (approximate)(m)

$L$ : Given distance $(\mathrm{m})$

$l_{i}$ : Given distance on sensors $(\mathrm{m})$

$f:$ Focal length (approximate) $(\mathrm{m})$

Therefore, the relative distance between the lowest principal point and the other $B_{z 1}, B_{z 2}, \ldots$ and $B_{z 5}$ could be calculated by the following equation.

$B_{z i}=H_{i}-H_{1} \quad(i=1,2,3, \Lambda)$

where,

$B_{z i}:$ Distance between principal points (approximate) (m)

$H_{i}:$ Altitude of pictures (approximate) (m)

\subsection{Relative Orientation}

Generally, relative orientation is applied to a pair of images (Wang et al., 2012). In this paper, the image at the lowest altitude was set as a basis image, and the coplanarity condition was applied between the basis image and other images. In figure 6, it is assumed that image No. 1 was taken at the origin and not rotated, and relative positions and rotation angles of the other images were calculated simultaneously. Moreover, the interior 


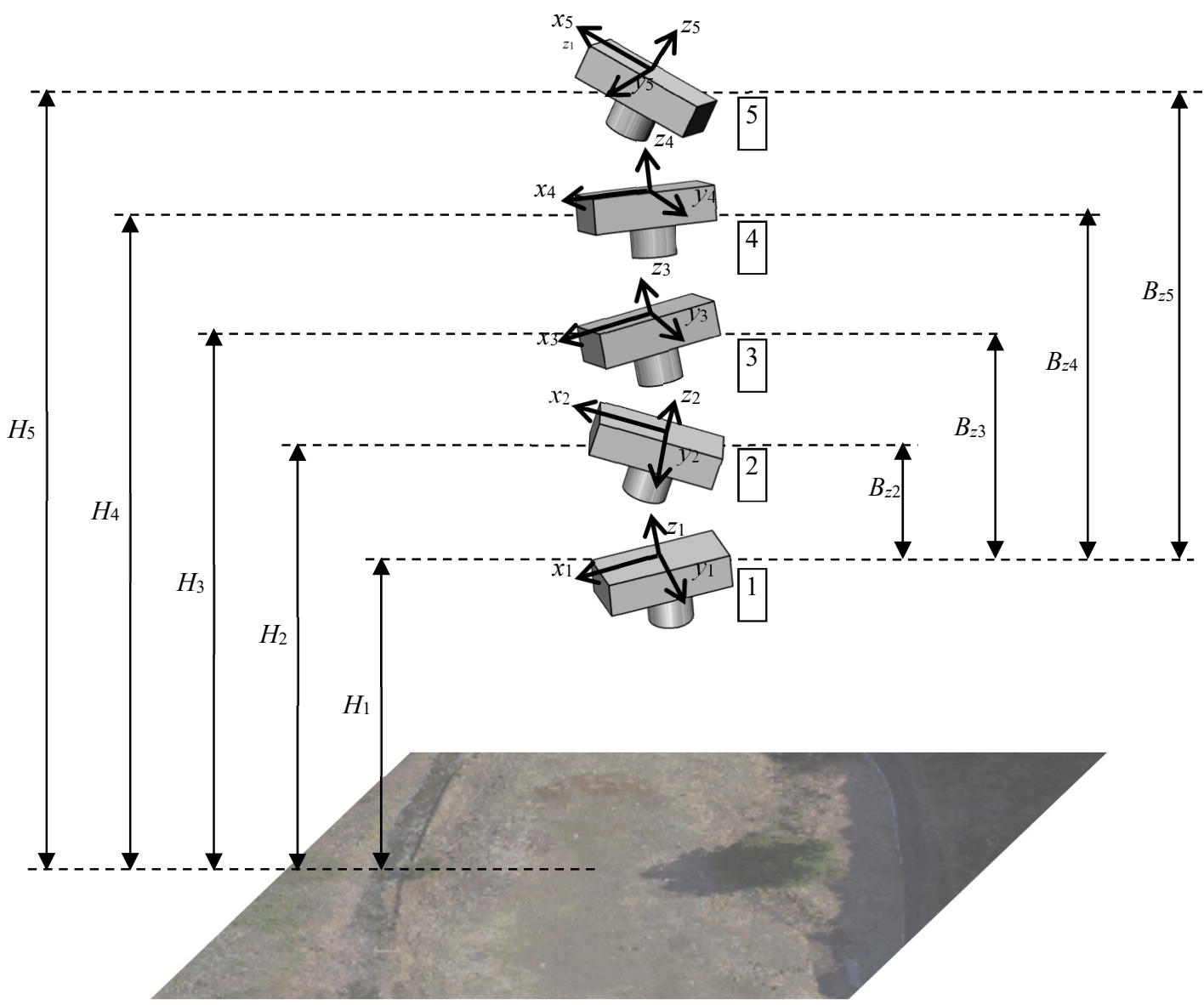

Figure 6. Positional relation of vertical images

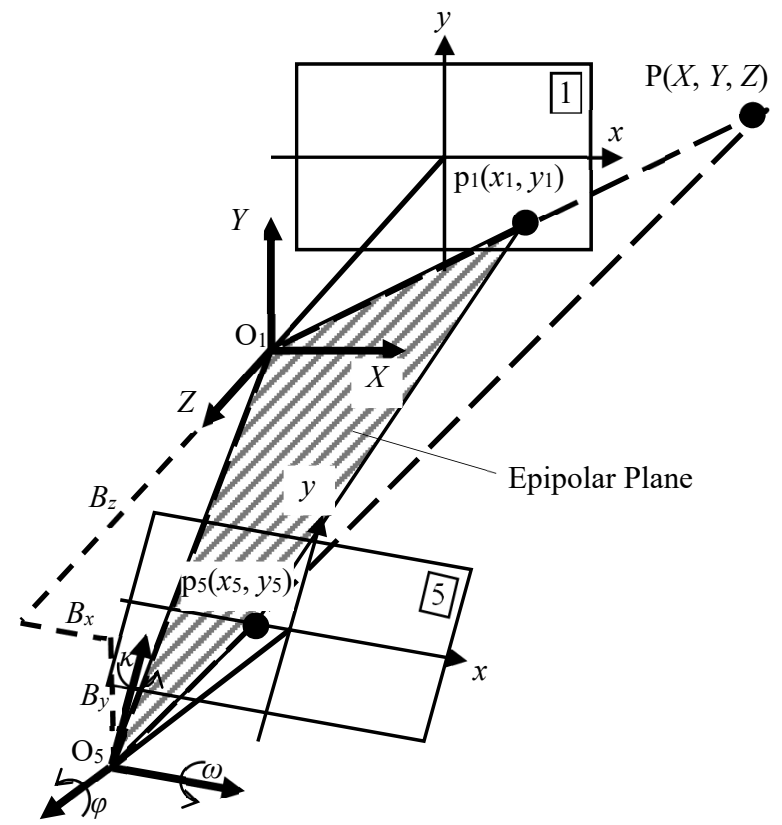

Figure 7. Coplanarity condition of two vertical images

parameters of the camera were set as unknown parameters and were also calculated by this orientation.
Figure 7 shows coplanarity condition for images No. 1 and No. 5 of those vertical images. The epipolar plane was constituted by two principal points and a corresponding point $\mathrm{P}$. Let the principal points of each image be $\mathrm{O}_{1}(0,0,0)$ and $\mathrm{O}_{5}\left(B_{x}, B_{y}, B_{z}\right)$, image points of $\mathrm{P}$ be $\mathrm{p}_{1}\left(x_{1}, y_{1}\right)$ and $\mathrm{p}_{5}\left(x_{5}, y_{5}\right)$. Then, the relationship of these two images is expressed by the following coplanarity equation.

$$
\left|\begin{array}{ccc}
B_{x} & B_{y} & B_{z} \\
X_{1} & Y_{1} & Z_{1} \\
X_{5} & Y_{5} & Z_{5}
\end{array}\right|=0
$$

where,

$$
\begin{aligned}
& \left(\begin{array}{c}
X_{1} \\
Y_{1} \\
Z_{1}
\end{array}\right)=\left(\begin{array}{c}
x_{1} \\
y_{1} \\
-f
\end{array}\right) \\
& \left(\begin{array}{c}
X_{5} \\
Y_{5} \\
Z_{5}
\end{array}\right)=\mathbf{R}\left(\begin{array}{c}
x_{5} \\
y_{5} \\
-f
\end{array}\right)+\left(\begin{array}{l}
B_{x} \\
B_{y} \\
B_{z}
\end{array}\right)
\end{aligned}
$$$$
\mathbf{R}=\left(\begin{array}{ccc}
1 & 0 & 0 \\
0 & \cos \omega & -\sin \omega \\
0 & \sin \omega & \cos \omega
\end{array}\right)\left(\begin{array}{ccc}
\cos \varphi & 0 & \sin \varphi \\
0 & 1 & 0 \\
-\sin \varphi & 0 & \cos \varphi
\end{array}\right)\left(\begin{array}{ccc}
\cos \kappa & -\sin \kappa & 0 \\
\sin \kappa & \cos \kappa & 0 \\
0 & 0 & 1
\end{array}\right)
$$
$\omega, \varphi, \kappa:$ rotation angle of No.5

$f$ : focal length 
Table 1. Unknown parameters of relative orientation

\begin{tabular}{|c|c|c|c|}
\hline \multicolumn{2}{|c|}{$\begin{array}{l}\text { Interior Parameters } \\
\text { (10) }\end{array}$} & \multicolumn{2}{|c|}{$\begin{array}{l}\text { Relative Orientation Parameters } \\
\qquad(5 \times(n-1))\end{array}$} \\
\hline Focal Length (mm) & $f$ & & $\omega_{2}, \omega_{3}, \omega_{4}, \ldots$ \\
\hline Principal Point (pixel) & $x_{p}, y_{p}$ & Rotation Angle & $\varphi_{2}, \varphi_{3}, \varphi_{4}, \ldots$ \\
\hline Scale Factor & $a_{1}, a_{2}$ & & $\kappa_{2}, \kappa_{3}, \kappa_{4}, \ldots$ \\
\hline $\begin{array}{l}\text { Coefficients of } \\
\text { Radial Distortion }\end{array}$ & $k_{1}, k_{2}, k_{3}$ & acition & $B_{x 2}, B_{x 3}, B_{x 4}, \ldots$ \\
\hline $\begin{array}{l}\text { Coefficients of } \\
\text { Tangential Distortion }\end{array}$ & $p_{1}, p_{2}$ & 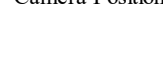 & $B_{y 2}, B_{y 3}, B_{y 4}, \ldots$ \\
\hline
\end{tabular}

$B_{z}$ in the coplanarity equation is substituted by $B_{z i}$ in equation 2 . Therefore, $B_{z}$ is set as a fixed value in this coplanarity condition, and the relative orientation is performed. In addition, the interior parameters are also set as unknown parameters in this relative orientation and are shown in table 1. Therefore, the image coordinates of the $\mathrm{p}_{1}$ and $\mathrm{p}_{5}$ should contain the interior parameters in equation 3 . In this condition, $x_{i}$ and $y_{i}(i=1,5)$ are calculated by following equations:

$\left.\begin{array}{l}x_{i}=x_{i}^{\prime}+x_{i}^{\prime}\left(k_{1} r^{2}+k_{2} r^{4}+k_{3} r^{6}\right)+p_{1}\left(r^{2}+2 x_{i}^{\prime 2}\right)+2 p_{2} x_{i}^{\prime} y_{i}^{\prime} \\ y_{i}=y_{i}^{\prime}+y_{i}^{\prime}\left(k_{1} r^{2}+k_{2} r^{4}+k_{3} r^{6}\right)+p_{2}\left(r^{2}+2 y_{i}^{\prime 2}\right)+2 p_{1} x_{i}^{\prime} y_{i}^{\prime}\end{array}\right\}$

where,

$k_{1}, k_{2}, k_{3}$ : Coefficients of radial distortion

$p_{1}, p_{2}$ : Coefficients of tangential distortion

$r=\sqrt{x_{i}^{\prime 2}+y_{i}^{\prime 2}}$

$u_{i}=x_{p}+a_{1} x_{i}^{\prime}+a_{2} y_{i}^{\prime}$

$v_{i}=y_{p}+a_{3} x^{\prime}+a_{4} y_{i}^{\prime}$

$x_{i}^{\prime}, y_{i}^{\prime}$ : Measurement point (mm)

$u_{i}, v_{i}:$ Measurement point (pixel)

$x_{p}, y_{p}:$ Principal point (pixel)

$a_{1}, a_{2}, a_{3}, a_{4}:$ Scale factor
Consequently, the coplanarity equations were acquired between image No.1 image and other images respectively. In order to calculate these unknown parameters shown in table 1, a sufficient number of tie points of every image for acquisition of coplanarity equations is required. For example, the number of unknown parameters in the case of five images is $10+5 \times(5-1)=30$. On the other hand, if over eight tie points can be obtained, the number of coplanarity equations is more than $8 \times(5-1)=32$. These tie points are used as measurement points in this paper.

\subsection{Calculation of 3D Coordinates}

The relative orientation parameters and the interior parameters of all images can be acquired. The 3D relative coordinates of every measurement point are calculated by collinearity condition. Each measurement point in an image gives two collinearity equations. Therefore, the number of collinearity equations in the case of five images is $2 \times 5=10$. Consequently, the three unknown parameters of the 3D relative coordinates can be calculated by collinearity equations, and the $3 \mathrm{D}$ relative coordinates for every measurement point can be acquired. These $3 \mathrm{D}$ relative coordinates are converted to absolute coordinates by using the given distance shown in figure 5 . The conversion is performed by the ratio of the given distance between the absolute value and the pixel value. In addition, the origin point and the three axes of the absolute coordinate should be set respectively.

\subsection{Absolute Orientation}

The absolute orientation which can calculate the interior and the exterior parameters is performed by using the absolute $3 \mathrm{D}$ coordinate of the measurement points. Every measurement point is set as a GCP, and the common interior parameters and exterior parameters of every image are calculated simultaneously by bundle adjustment contained in collinearity equations. As shown in table 1 , the number of common interior parameters is ten. The number of exterior parameters for each image is six, which are camera positions and rotation angles. Therefore, the number of unknown parameters for this absolute orientation in the case of five images is $10+6 \times 5=40$. For such parameters, ten collinearity equations can be acquired for one measurement point. Consequently, every unknown parameter can be calculated by four or more measurement points, and the absolute orientation can be achieved.

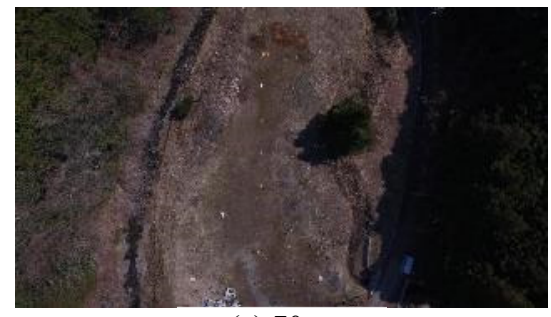

(a) $70 \mathrm{~m}$

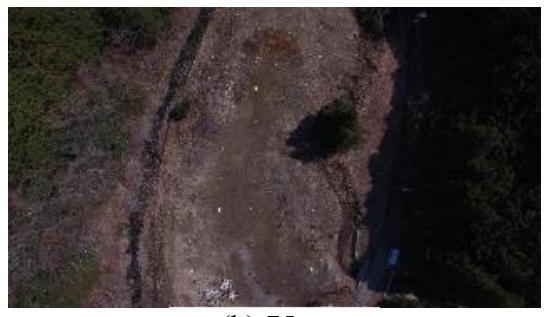

(b) $75 \mathrm{~m}$

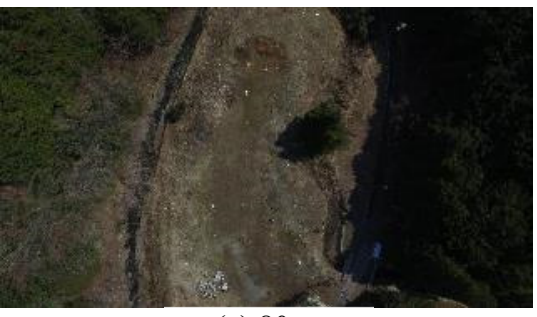

(c) $80 \mathrm{~m}$

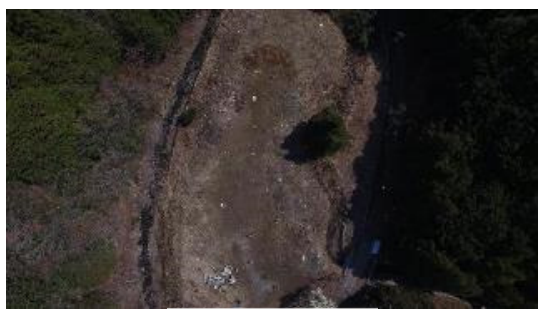

(d) $85 \mathrm{~m}$

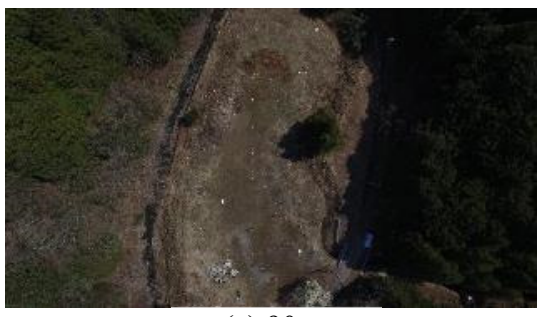

(e) $90 \mathrm{~m}$

Figure 8. Vertical images for checking accuracy 


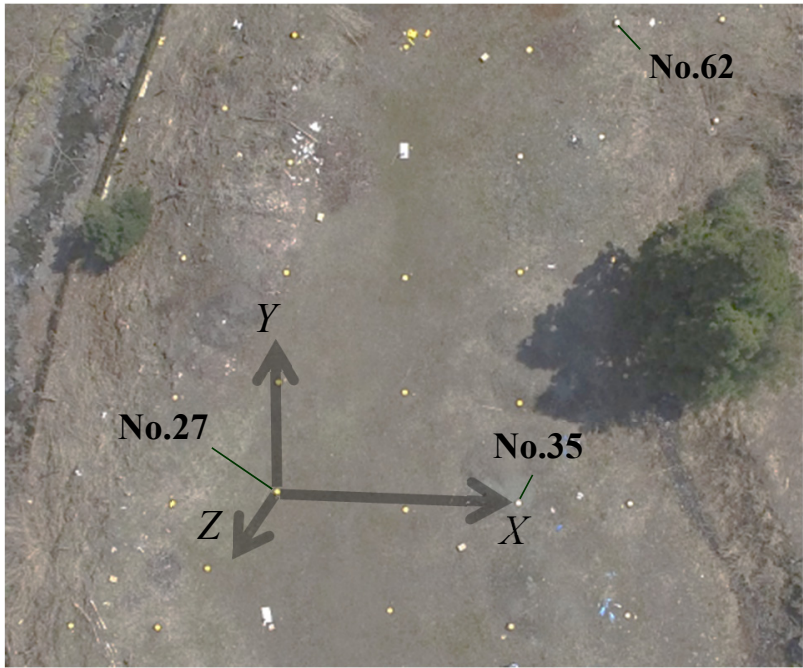

Figure 9. Local coordinate system

\subsection{Final Orientation}

The orientation parameters for every camera and the absolute 3D coordinates for every measurement point were acquired by the procedure described above. However, errors in estimation of the absolute 3D coordinates are possible due to conversion from the relative coordinates if using only one given distance. Therefore, the final orientation is performed by using every orientation parameter and every 3D coordinate of the measurement points, which are set as approximate values. By setting the collinearity equations from every GCP as observation equations, the final orientation is calculated.

\section{CHECKING ACCURACY}

\subsection{Images Taken at the UAV Test Site}

In order to evaluate the performance of the proposed method, the accuracy of the measurement was checked by using the vertical images taken at the UAV test site. For taking the images, the UAV was operated to fly vertically and the images were taken at every $5 \mathrm{~m}$ altitude between $70 \mathrm{~m}$ to $90 \mathrm{~m}$. Figure 8 shows the five taken images. All 39 ground marks were included in all five images and set as check points for checking the accuracy of the 3D coordinates. Figure 9 shows the local coordinate system for checking the accuracy. The ground mark No. 27 was set as the origin point and the direction to No. 35 was set as $X$ axis. The plane made of these two points and No. 62 was set as X-Y plane; the orthogonal direction of the $\mathrm{X}-\mathrm{Y}$ plane was set as $\mathrm{Z}$ axis.

The accuracy of the proposed method was also compared with Agisoft PhotoScan, which is one of the SfM software. The photogrammetry by PhotoScan was attempted by three methods. In the first method, the five vertical images we used and the three points for the decision of axes were set as GCPs. In addition, other 36 points were set as check points for accuracy evaluation. In the second method, the same five images were used, and all 39 points were set as GCPs. The accuracy was evaluated by errors of the GCPs. In the third method, 57 general photogrammetric images were used. The UAV was moved horizontally at about $70 \mathrm{~m}$ fixed altitude and the overall UAV test site was taken with an $80 \%$ overlapping rate between the images. In addition, nine ground marks were set as GCPs, and other 30 points were set as check points for accuracy evaluation.

In order to evaluate the accuracy of each method quantitatively, the standard error was also calculated by following equation (Yanagi and Chikatsu, 2016).
$\sigma_{x}=\sigma_{y}=\frac{H}{f} \sigma_{p}, \quad \sigma_{z}=\sqrt{2} \frac{H}{f} \frac{H}{B} \sigma_{p}$

where,

$\sigma_{x}, \sigma_{y}, \sigma_{z}:$ Standard error for each axis (m)

$H:$ Altitude (m)

$f:$ Focal length $(\mathrm{m})$

$B:$ Base line (m)

$\sigma_{p}:$ Pointing accuracy $(\mathrm{m})$

In the above equation, $H$ was set as an average value of five vertical images $(83.944 \mathrm{~m})$ and $B$ was set as a maximum distance between two images out of five $(20.137 \mathrm{~m})$. The pointing accuracy was set as a length of one pixel on the sensor.

\subsection{Results of Accuracy Checking}

Table 2 shows the results of the final orientation and table 3 shows the results of checking the accuracy. The root-mean-square errors (RMSE) of X, Y, and Z accuracies were calculated to be within $\pm 0.200 \mathrm{~m}$. These accuracies are acceptable for surveying a construction site.

The PhotoScan results are shown in the same table. The results of five vertical images with 39 GCPs were similar in value to the results in the proposed method. Moreover, compared to the standard accuracy, $Z$ accuracy of the proposed method was higher. Therefore, it can be said that the proposed method is more efficient as it does not employ the GCPs. Still, the highest accuracy was obtained by the general photogrammetric method which uses horizontal images. Consequently, the UAV imagetaking method should be selected according to the situation in the measurement field. Nevertheless, the proposed method can be a convenient and efficient measurement method under certain circumstances.

Table 2. Results of final orientation

\begin{tabular}{|c|c|c|c|c|c|c|}
\hline & Altitude & $70 \mathrm{~m}$ & $75 \mathrm{~m}$ & $80 \mathrm{~m}$ & $85 \mathrm{~m}$ & $90 \mathrm{~m}$ \\
\hline \multirow{3}{*}{$\begin{array}{l}\text { Camera } \\
\text { position }\end{array}$} & $X_{0}(\mathrm{~m})$ & 13.648 & 13.823 & 14.302 & 14.180 & 14.325 \\
\hline & $Y_{0}(\mathrm{~m})$ & -0.619 & -1.052 & -1.297 & -1.528 & -1.958 \\
\hline & $Z_{0}(\mathrm{~m})$ & 74.061 & 78.676 & 83.806 & 89.035 & 94.142 \\
\hline \multirow{3}{*}{$\begin{array}{c}\text { Rotation } \\
\text { angle }\end{array}$} & $\omega$ & $6^{\prime} \quad 58^{\prime \prime}$ & $6^{\prime} \quad 43^{\prime \prime}$ & $6^{\prime} \quad 35^{\prime \prime}$ & $6^{\prime} 33^{\prime \prime}$ & $6^{\prime} \quad 28^{\prime \prime}$ \\
\hline & $\varphi$ & $1^{\prime} \quad 12^{\prime \prime}$ & $1^{\prime} \quad 15^{\prime \prime}$ & $1^{\prime} \quad 10^{\prime \prime}$ & $1^{\prime} \quad 09^{\prime \prime}$ & $0^{\prime} \quad 59^{\prime \prime}$ \\
\hline & $\kappa$ & $3^{\prime} \quad 15^{\prime \prime}$ & $3^{\prime} \quad 00^{\prime \prime}$ & $3^{\prime} \quad 00^{\prime \prime}$ & $3^{\prime} 14^{\prime \prime}$ & $2^{\prime} 58^{\prime \prime}$ \\
\hline
\end{tabular}

Table 3. Results of checking accuracy

\begin{tabular}{|c|c|c|c|c|}
\hline \multirow{2}{*}{\multicolumn{2}{|c|}{ Measurement method }} & \multicolumn{3}{|c|}{ RMSE (m) } \\
\hline & & $\mathrm{X}$ & $\mathrm{Y}$ & Z \\
\hline \multicolumn{2}{|c|}{$\begin{array}{l}\text { Standard accuracy for } \\
5 \text { vertical images }\end{array}$} & \pm 0.040 & \pm 0.040 & \pm 0.233 \\
\hline $\begin{array}{l}\text { Proposal } \\
\text { method }\end{array}$ & $\begin{array}{l}5 \text { vertical images, } \\
\text { no GCPs }\end{array}$ & \pm 0.096 & \pm 0.160 & \pm 0.166 \\
\hline \multirow{3}{*}{ PhotoScan } & $\begin{array}{l}5 \text { vertical images, } \\
3 \text { GCPs }\end{array}$ & \pm 4.808 & \pm 3.437 & \pm 10.109 \\
\hline & $\begin{array}{l}5 \text { vertical images, } \\
39 \text { GCPs }\end{array}$ & \pm 0.094 & \pm 0.098 & \pm 0.574 \\
\hline & $\begin{array}{l}57 \text { horizontal images, } \\
9 \mathrm{GCPs}\end{array}$ & \pm 0.038 & \pm 0.030 & \pm 0.048 \\
\hline
\end{tabular}




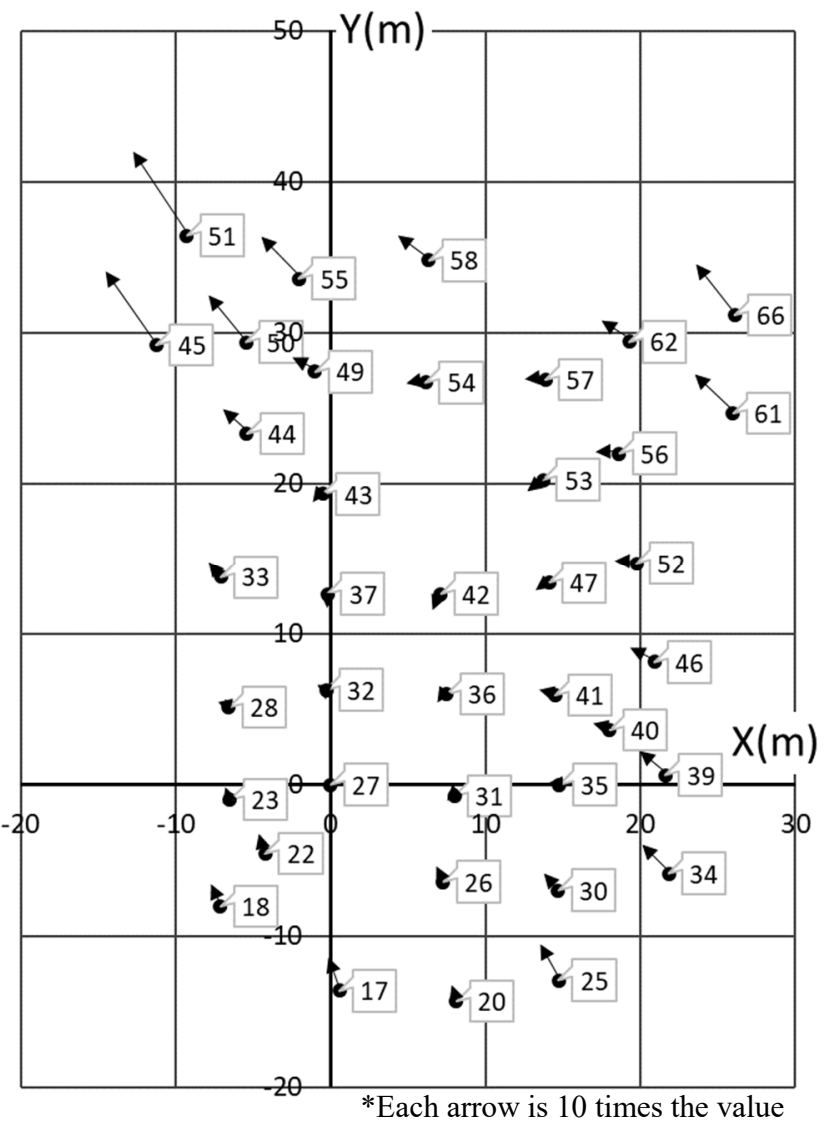

Figure 10. Error distribution of the proposed method

Figure 10 shows the error distribution of the measured $\mathrm{X}$ and $\mathrm{Y}$ coordinates for the proposed method. The errors at the points around the origin are smaller than those at the distant points. In particular, the errors of No. 45, 50, 51, 55, 58, 61, and 66 are indicated as over $\pm 0.3 \mathrm{~m}$. On the other hand, the distribution of the measurement points for $\mathrm{Y}$ direction was wider than for $\mathrm{X}$ direction. In addition, the $Y$ accuracy of the proposed method was lower than the $\mathrm{X}$ accuracy, as shown in table 3. Therefore, the accuracies of such distant points along the $\mathrm{Y}$ direction were influenced more than in the $\mathrm{X}$ direction. For that reason, the origin point should be set as close to an important area in the measurement field as possible.

\section{CONCLUSIONS}

A measurement method which requires a simple flight of a UAV and a few vertical images was developed. The orientation in this method can be performed by using only one distance, without using the GCPs. In order to evaluate the performance of the proposed method, the accuracy of the proposed method was compared with some traditional methods by PhotoScan. The proposed method uses only a small number of vertical images and a lot less labour and skills compared to the traditional methods. The accuracy of the proposed method was calculated. The method was confirmed as applicable for construction sites. It can also be used in natural disaster fields thanks to its accuracy and efficiency.

As further work, some techniques for improving accuracy should be considered. In particular, the accuracy of a distant-from-theorigin point should be improved. A suitable number of images, suitable altitude, and the use of the global navigation satellite system (GNSS) for positioning should be investigated.

\section{ACKNOWLEDGEMENTS}

This investigation was supported by JSPS KAKENHI Grant Number 16K08133. I would like to offer my special thanks to the Test Field Committee of the Japan Society of Photogrammetry and Remote Sensing.

\section{REFERENCES}

Amrullah, C., Suwardhi, D., Meilano, I., 2016. Product Accuracy Effect of Oblique and Vertical Non-Metric Digital Camera Utilization in UAV-Photogrammetry to Determine Fault Plane. In: International Archives of the Photogrammetry, Remote Sensing and Spatial Information Sciences, Prague, Czech Republic, Vol. XLI-B1, pp.41-48.

Bagheri, O., Ghodsian, M., Saadatseresht, M. 2015. Reach Scale Application of UAV+SfM Method in Shallow Rivers Hyperspatial Bathymetry. In: International Archives of the Photogrammetry, Remote Sensing and Spatial Information Sciences, Kish Island, Iran, Vol. XL-1/W5, pp.77-81.

Barazzetti, L., Brumana, R., Oreni, D., Previtali, M., Roncoroni, F., 2014. True-Orthophoto Generation from UAV Images: Implementation of a Combined Photogrammetric and Computer Vision Approach. In: ISPRS Annals of the Photogrammetry, Remote Sensing and Spatial Information Sciences, Riva del Garda, Italy, Vol. II-5, pp.77-81.

Beaudoin, L., Avanthey, L., Gademer, A., Roux, M., Rudant, J., 2015. Dedicated Payloads for Low Altitude Remote Sensing in Natural Environments. In: International Archives of the Photogrammetry, Remote Sensing and Spatial Information Sciences, La Grande Motte, France, Vol. XL-3/W3, pp.405-410.

Feifei, X., Zongjian, L., Dezhu, G., Huad, L., 2012. Study on Construction of 3D Building Based on UAV Images. In: International Archives of the Photogrammetry, Remote Sensing and Spatial Information Sciences, Melbourne, Australia, Vol. XXXIX-B1, pp.469-473.

Galarreta, J., Kerle, N., Gerke, M., 2015. UAV-Based Urban Structural Damage Assessment using Object-Based Image Analysis and Semantic Reasoning. In: Natural Hazards Earth System Sciences., Vol.15, pp.1087-1101, doi:10.5194/nhess-151087-2015

Li, M., Li, D, Fanb, D., 2012. A Study on Automatic UAV Image Mosaic Method for Paroxysmal Disaster. In: International Archives of the Photogrammetry, Remote Sensing and Spatial Information Sciences, Melbourne, Australia, Vol. XXXIX-B6, pp.123-128.

Longhitano, G., Quintanilha, J., 2013. Rapid Acquisition of Environmental Information After Accidents with Hazardous Cargo Through Remote Sensing by UAV. In: International Archives of the Photogrammetry, Remote Sensing and Spatial Information Sciences, Hannover, Germany, Vol. XL-1/W1, pp.201-205.

Persad, R., Armenakis, C., 2016. Co-Registration of DSMs Generated by UAV and Terrestrial Laserscanning Systems. In: International Archives of the Photogrammetry, Remote Sensing and Spatial Information Sciences, Prague, Czech Republic, Vol. XLI-B1, pp.985-990. 
Tanzi. T., Chandra. M., Isnard, J., Camara, D., Sebastien, O., Harivelo, F., 2016. Towards "Drone-Borne" Disaster

Management: Future Application Scenarios. In: ISPRS Annals

of the Photogrammetry, Remote Sensing and Spatial

Information Sciences, Prague, Czech Republic, Vol. III-8, pp.181-189.

Valavanis, K., Vachtsevanos, G., 2015. Handbook of Unmanned Aerial Vehicles. Springer Netherlands, https://doi.org/10.1007/978-90-481-9707-1

Wang, J., Lin, Z., Ren, C., 2012. Relative Orientation in Low Altitude Photogrammetry Survey. In: International Archives of the Photogrammetry, Remote Sensing and Spatial Information Sciences, Melbourne, Australia, Vol. XXXIX-B1, pp.463-467.

Westoby, J., Brasinton, J., Glasser F., Hambrey J., Reynolds M., 2012. 'Structure-from-Motion' Photogrammetry: A Lowcost, Effective Tool for Geoscience Applications, Geomorphology, Vol. 179, pp.300-314.

Yanagi, H., Chikatsu, H., 2016. Performance Evaluation of 3D Modeling Software for UAV Photogrammetry. In: International Archives of the Photogrammetry, Remote Sensing and Spatial Information Sciences, Prague, Czech Republic, Vol. XLI-B5, pp.147-152. 